\title{
FACE ReCOgnition From A Single SAMPLE USING RLOg FILTER AND MANifOLd ANALYSIS
}

\author{
Jaya Susan Edith. $\mathrm{S}^{1}$ and A.Usha Ruby ${ }^{2}$ \\ ${ }^{1}$ Department of Computer Science and Engineering,CSI College of Engineering, \\ ${ }^{2}$ Research scholar, Bharath University
}

\begin{abstract}
Face recognition is A technique that has been widely used in various important field, this process helps in the identification of an individual by a machine for the purpose of security and ease of work. The normal technique of face recognition usually works better when there are multiple samples for a single person (MSSP) is available. In present applications where this technique is to be used such as in social networks, security systems, identification cards there is only a single sample per person (SSPP) that is readily available. This less availability of the samples causes failure in the working of conventional face recognition techniques which require multiple samples for a particular individual. To overcome this drawback which sets back the system from the accurate functioning of face recognition this paper puts forward a novel technique which makes use of discriminative multi-manifold analysis (DMMA) that extracts distinctive features using image patches. Recognition is done by the process of manifold to manifold matching. Hence there is an increment in the accuracy rate of face recognition.
\end{abstract}

\section{KEYWORDS}

Face recognition; manifold learning; filter; clustering.

\section{INTRODUCTION}

Face recognition is a process in which a machine system automatically recognizes an individual from a digital image or video based on formerly stored samples of that individual. It is more beneficial and easier to use than other biometric techniques used for security such as fingerprint or iris recognition, because it can be done passively without any intervention of the person which is a must for other techniques for the purpose of identification of the individual. Many techniques are currently being used for the face recognition out of which one is to associate extracted facial features with the facial database. A large number of algorithms have been proposed, where the main goal is to learn in a supervised, semi-supervised or in unsupervised manner. The two primary tasks for which face recognition has been used are verification and identification. And its various applications include security, surveillances, general identity verification, criminal justice systems, and other similar application.

Single image per person consists of frontal face image; there are two eyes, one nose, and one mouth in the image. When comparison is done between eyes of two different persons the rate of similarity in appearance is higher when compared to the eyes and nose of the same person. When the image is made discrete into several local patches, it is more likely that these patches reside in 
a manifold and each patch corresponds to a point in the manifold. Manifold is a collection of similar datasets. The overlapping between the patches of dissimilar persons is more because their similarity is high and there is distance between the patches of one person. In order to increase the distance between dissimilar individuals manifold margins discriminative multimanifold analysis (DMMA) method is used.

\section{RELATED WORK}

To overcome the difficultly faced in using single sample per person (SSPP) for face recognition, there have been several attempts and they are categorized into four basic categories they are; local feature representations, generic learning, virtual sample generation, and image partitioning. In local feature representation feature descriptor is applied to represent each face image as a feature vector, and different persons features are expected to be separated from each other. In generic training sets multiple samples per person are used to extract the discriminative features which are used for the separation. For virtual sample generation each person's images are virtually generated. And image partitioning the sample image is portioned into multiple local patches used for feature extraction.

\section{PROPOSED APPROACH}

This paper makes use of interest point detection for the purpose of feature extraction followed by DMMA technique for the purpose of clustering all the points that are belonging to same individual into a single group. Interest point detection is done with the help of RLOG (Rank order Laplacian of Gaussian) filter which generates random interest points that help in the identification and feature extraction of an individual. ROLG filter has the following advantages in detecting interest points:

1. The light but strong structures has less influence on the output of the ROLG filter.

2. Structures, which incompletely fall in a detection window, have limited influence on the response of the ROLG filter.

3.Only one peak is created at the center of a blob, and no ring is generated around the blob.

4. The ROLG filter suppresses the output of edges. Therefore, no point is detected along edges.

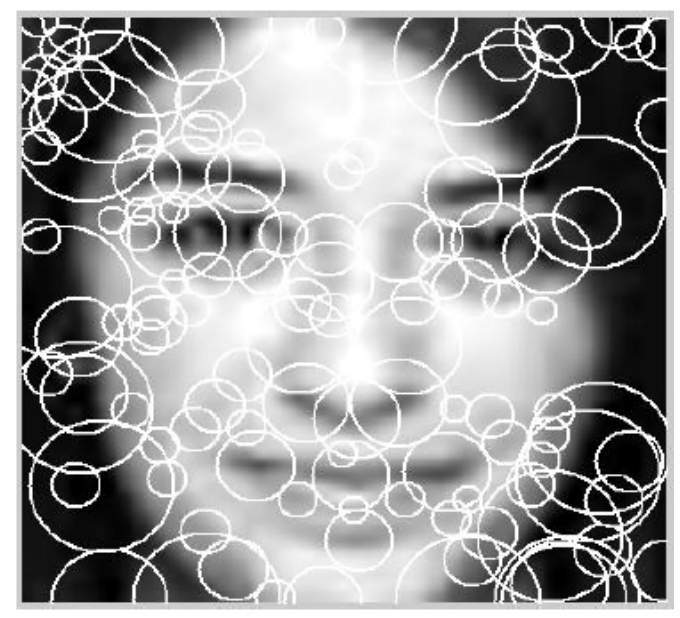

Figure 1. Interest point detection using RLOG filter. 


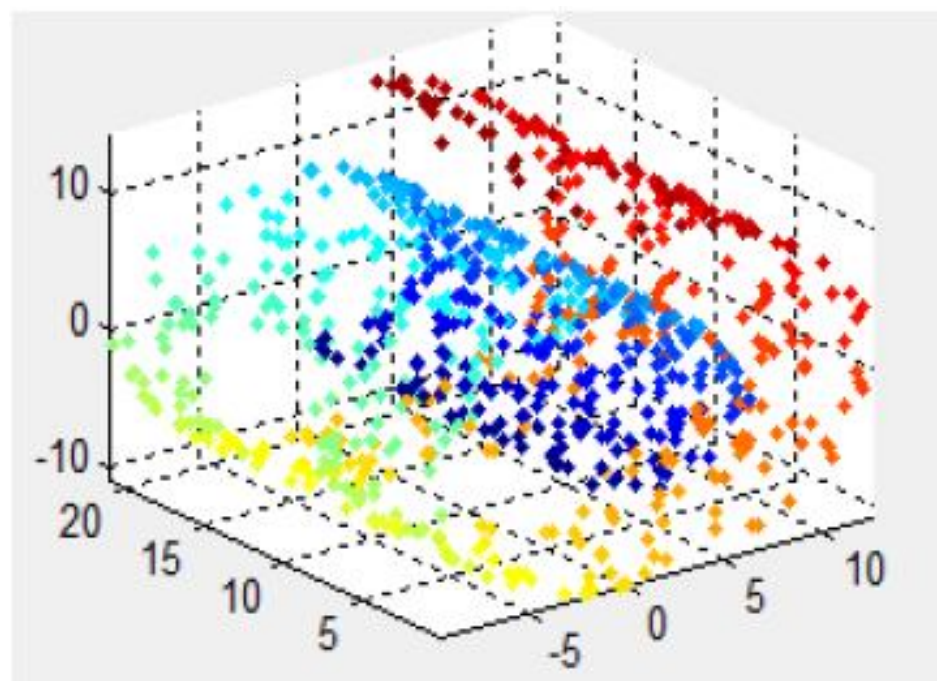

Fig. 2. Overlapping of patches of different persons.

The overlapping of patches is seen in figure 2 because eyes of two different people are similar in shape and texture when compared to the eyes and nose of the same person. The feature patches of the same individual are separated due to the dissimilarity in their appearance. To solve this problem DMMA is being used to separate the patches that do not belong to the same person and bring all the patches belonging to a single person into one manifold.

The main motive of using separate manifolds for different persons is that manifold for each person is person specific feature extraction method gain more accuracy than generic methods. The other reason is surviving techniques using manifold considers a single manifold for all faces and the measurement of feature of all face samples is preferred the same for recognition. The optimal feature dimension for each person may be different due to intrinsic differences of different persons. Due to this we model each person as a manifold so that we can optimize the feature dimensions for each individual.

Let $\mathrm{X}=[\mathrm{x} 1, \mathrm{x} 2, \ldots, \mathrm{xN}]$ be the training set, where $\mathrm{xj}$ is the training image of the jth person with the size of $m \times n, 1 \leq j \leq N, N$ is the number of persons in the training set. Each image $x j$ is partitioned image is into $t$ nonoverlapping feature patches with an equal size of ab where $t=\frac{\bar{m} \times m}{a \cdot b}$ .Let $\mathrm{Mi}=[\mathrm{xi} 1, \mathrm{xi} 2, \ldots ., \mathrm{xit}]$ be the image patch set of the ith person, represented by manifold Mi. Face is automatically detected using Viola-Jones face detector Each image is split into $20 \times 20$ nonoverlapping feature patches. The subjects are taken from database and the manifold structure is formed. The appearance of the distribution of all the patches in the manifold is depicted in figure 2. All patches of similar appearance are distributed together and the patches of one single individual are distributed far away from each other. To bring all the patches belonging one individual together DMMA technique is applied. 
Fig.3. Block diagram of face recognition system

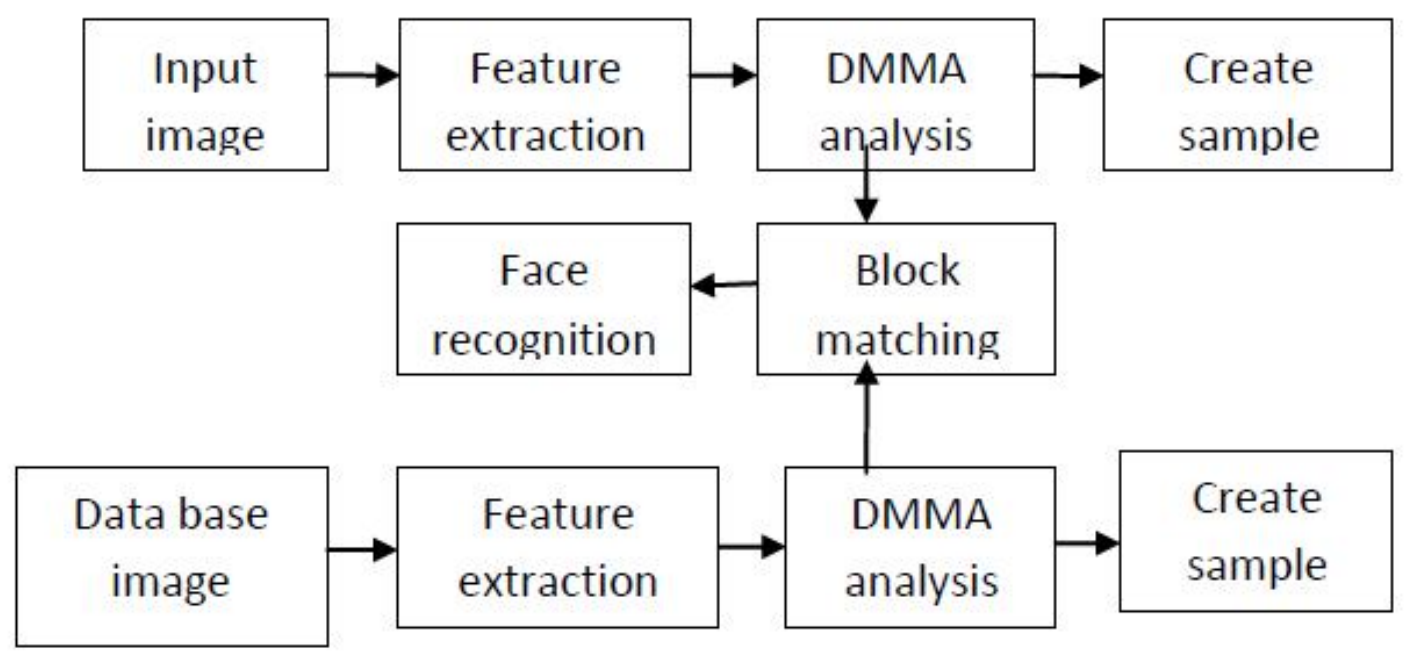

\section{DMMA method}

Let $\mathrm{M}=[\mathrm{M} 1, \mathrm{M} 2, \ldots, \mathrm{MN}]$ be the training set of $\mathrm{N}$ people and $\mathrm{Mi}=[\mathrm{xi1}, \mathrm{xi} 2, \ldots, \mathrm{xit}]$ be the manifold of the ith person, xir $€ \mathrm{Rd}, 1 \leq \mathrm{i} \leq \mathrm{t}$. DMMA seeks $\mathrm{N}$ feature matrices $\mathrm{W} 1, \mathrm{~W} 2$, ....,WN ,Wi Rd $\times$ di , $\mathrm{i}=1,2, \ldots, \mathrm{N}$, to project the training set into $\mathrm{N}$ low dimensional feature spaces in a way that the manifold margins are maximized, where $\mathrm{d}$ and di represent the feature dimension of each original image patch and the low dimensional feature learned by $\mathrm{Wi}$, respectively.

There are two types of neighbor present in this manifold for a sample manifold xir, which is the rth patch of the ith manifold as shown in figure 4: intermanifold neighbors Ninter and intramanifold neighbors Nintra. Intermanifold neighbors are the patches of same person and intramanifold neighbors are the patches of different persons. DMMA focuses on incrementing the manifold margins by reducing intermanifold distance and thereby incrementing the intramanifold distance.

$\max \mathrm{J}(\mathrm{W} 1, \mathrm{~W} 2, \ldots \mathrm{WN})$

$=\mathrm{J} 1(\mathrm{~W} 1, \mathrm{~W} 2, \ldots ., \mathrm{WN})-$

$\mathrm{J} 2(\mathrm{~W} 1, \mathrm{~W} 2, \ldots ., \mathrm{WN})$

$$
\begin{aligned}
& =\sum_{i=0}^{N}\left(\sum_{r=1}^{t} \sum_{p=1}^{k 1} \| \text { Wit xir }- \text { Wit xirp } \|^{2} \text { Airp }\right) \\
& -\sum_{i=0}^{N}\left(\sum_{r=1}^{t} \sum_{p=1}^{k 1} \| \text { Wit xir-Wit xirp } \|^{2} \text { Airp }\right)
\end{aligned}
$$


Where xirp represents the pth k1- nearest manifold and xirq denotes the qth k2- nearest intramanifold neighbor of xir .

There are two affinity matrix Airp and Birq to characterize the similarity between xirp and xir as well as that between xirq and xir.. The objective function of $\mathrm{J} 1$ is to ensure that if xir and xirp are close and from dissimilar subjects, then their low dimensional illustrations are separated as far as possible.On the other hand, J2 in ensures that if xir and xirq are close and from the same subject, then their low dimensional illustrations are close as well.

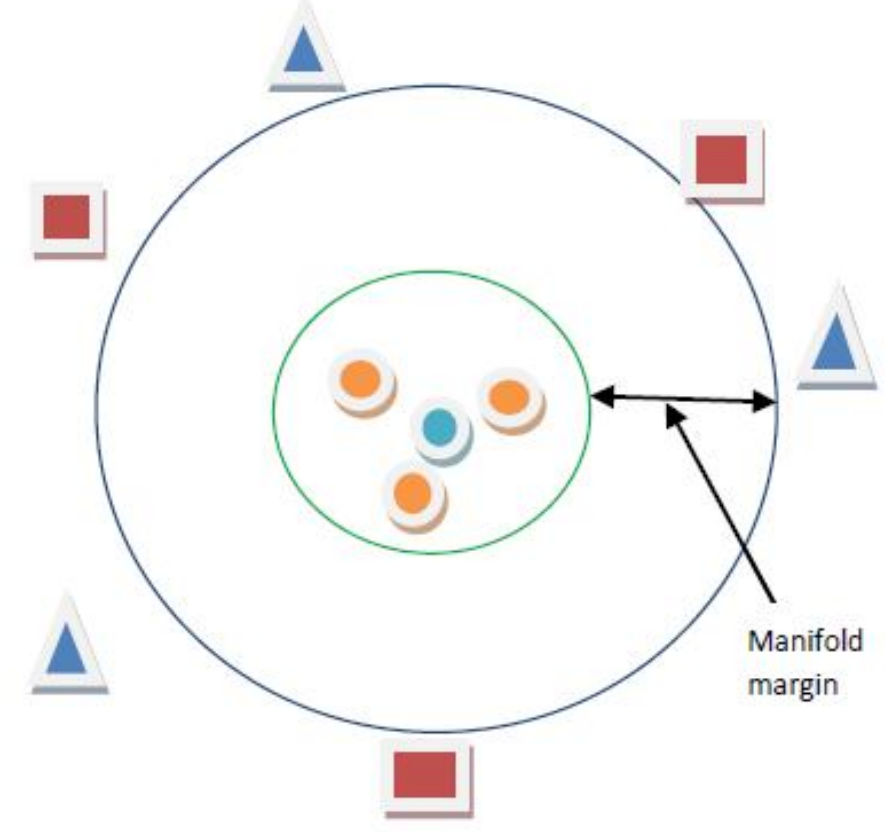

Fig. 4. There are six intermanifold neighbors present ouside and three intramanifold neighbor in the inner circle for local patch.

Procedure

Input: Manifold.

Output: Projection matrix.

Algorithm:

Step 1: Initialization.

Step 2: Similarity matrix calculation.

Step 3: Local optimization.

Step 4: Output Projection matrix. 


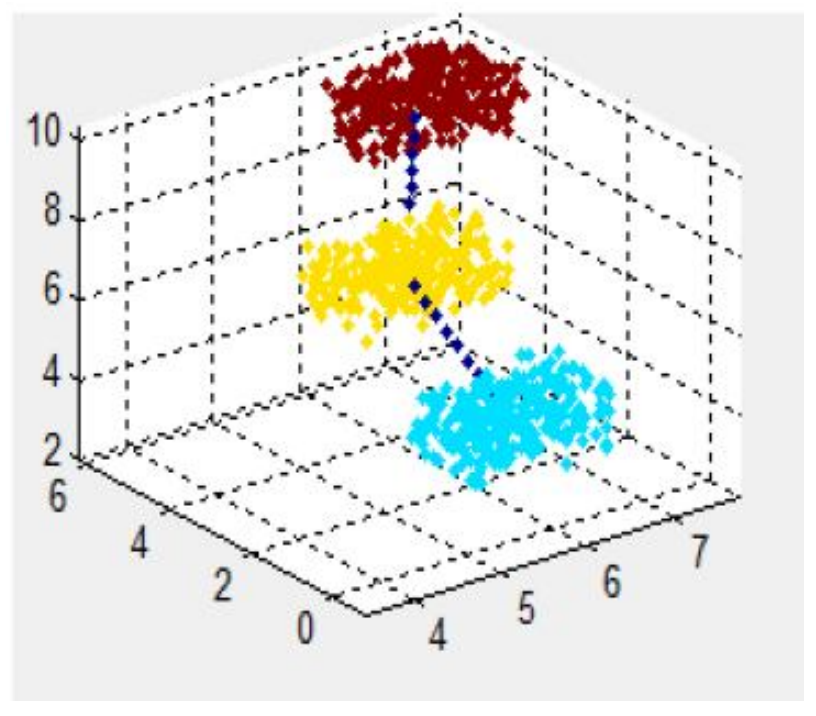

Fig. 5 Distribution of pixels after application of DMMA

We can select di eigenvectors to maximize . During the projection of specific eigenvector wj corresponding to an eigenvalue $\lambda_{\mathrm{j}}$ can be written as

$\mathrm{J}(\mathrm{wj})=\mathrm{J} 1(\mathrm{wj})-\mathrm{J} 2(\mathrm{wj})$

$=w j T(H 1-H 2) w j$

$={ }_{\mathrm{wj} T} \lambda_{\mathrm{wj}}$

$=\lambda_{\mathrm{j}}$

If $\lambda_{\mathrm{j}} 0$, it means the intermanifold distance is larger than the intramanifold distance along the direction of wj and the samples are correctly classified.

\section{CONCLUSION}

This paper introduces a novel high capacity face detection technology. In order to achieve a higher quality detecting system discriminative multi-manifold technique has been introduced. This technique makes use of only one image of a person stored in the database and this image is split into various patches, based on features the matching is done. The best matching feature is matched to the individual in the database hence this technique saves space and time.

\section{REFERENCES}

[1] Jiwen Lu, Member, IEEE, Yap-Peng Tan, Senior Member, IEEE, and Gang Wang, Member, IEEE, Discriminative Multimanifold Analysis for Face Recognition from a Single Training Sample per Personll. IEEE transactions on pattern analysis and machine intelligence, vol. 35, no. 1, January 2013.

[2] H. Hu, - Orthogonal Neighborhood Preserving Discriminant Analysis for Face Recognition,॥ Pattern Recognition, vol. 41, No. 6, pp. 2045-2054, 2008.

[3] A. Lanitis, -Evaluating the Performance of Face-Aging Algorithms,\|Proc. IEEE Int'l Conf. Automatic Face and Gesture Recognition, pp. 1-6, 2008. 
[4] S. Yan, D. Xu, B. Zhang, H. Zhang, Q. Yang, and S. Lin, -Graph Embedding and Extensions: A General Framework for Dimensionality Reduction,॥ IEEE Trans. Pattern Analysis and Machine Intelligence, vol. 29, no. 1, pp. 40-51, Jan. 2007.

[5] R. Wang and X. Chen. \|Manifold Discriminant Analysis\|l. In IEEE Conference on Computer Vision and Pattern Recognition, pages 1-8, 2009.

[7] R. Wang, S. Shan, X. Chen, and W. Gao, -Manifold-Manifold Distance with Application to Face Recognition Based on Image Set,\| Proc. IEEE Conf. Computer Vision and Pattern Recognition, pp. 18, 2008.

[8] D. Zhang and Z. Zhou, -2D2PCA: Two-Directional Two-Dimensional PCA for Efficient Face Representation and Recognition,\|l Neurocomputing, vol. 69, nos. 1-3, pp. 224-231, 2005.

[9] Zhenwei Miao n, Xudong Jiang School of Electrical and Electronic Engineering, Nanyang Technological University, Nanyang Link, Singapore 639798 ,Singapore. 2013 Elsevier. 